EPJ Web of Conferences 37, 01024 (2012)

DOI: $10.1051 /$ epjconf/20123701024

(C) Owned by the authors, published by EDP Sciences, 2012

\title{
ABC Effect in Double-Pionic Fusion - a New Resonance?
}

\author{
A. Pricking ${ }^{1,2}$, M. Bashkanov ${ }^{1,2}$, H. Clement ${ }^{1,2, a}$, E. Doroshkevich ${ }^{1,2}$, E. Perez del Rio ${ }^{1,2}$,
}

T. Skorodko ${ }^{1,2}$, and G. J. Wagner ${ }^{1,2}$ for the WASA-at-COSY Collaboration

1 Physikalisches Institut, Univ. Tübingen, Auf der Morgenstelle 14, D-72076 Tübingen, Germany

2 Kepler Center for Astro and Particle Physics, Univ. Tübingen, Auf der Morgenstelle 14, D-72076 Tübingen

\begin{abstract}
The $\mathrm{ABC}$ effect, an intriguing low-mass enhancement in the $\pi \pi$ invariant mass spectrum - known since more than 50 years from inclusive measurements of double-pionic fusion reactions - is reexamined. To this end exclusive and kinematically complete high-statistics experiments of the fusion reactions to d, ${ }^{3} \mathrm{He}$ and ${ }^{4} \mathrm{He}$ have been carried out with WASA at COSY. These measurements cover the full energy region, where the $\mathrm{ABC}$ effect has been observed previously. They also complement the systematic measurements of nucleon-nucleon induced two-pion production. An isospin decomposition of all three basic double-pionic fusion reactions leading to the deuteron uniquely shows that solely the isoscalar reaction part exhibits the $\mathrm{ABC}$ effect tightly correlated with a narrow resonance structure in the total cross section. The peak energy of the resonance structure is about $90 \mathrm{MeV}$ below the nominal $\Delta \Delta$ threshold of $2 m_{\Delta}$ and its width of only $70 \mathrm{MeV}$ is much less than the $2 \Gamma_{\Delta}$ expected from the conventional $t$-channel $\Delta \Delta$ process. Based on angular distributions the quantum numbers $I\left(J^{P}\right)=0\left(3^{+}\right)$have been assigned. In the double-pionic fusion reaction $d d \rightarrow \rightarrow^{4} \mathrm{He} \pi^{0} \pi^{0}$ again the $\mathrm{ABC}$ effect is observed to be correlated with the appearance of a resonance-like structure in the total cross section at the same excess energy. From this we conclude that this resonance structure obviously is strong enough to survive even in nuclei.
\end{abstract}

\section{Introduction}

The $\mathrm{ABC}$ effect, an intriguing low-mass enhancement in the $\pi \pi$ invariant mass spectrum, has first been observed in inclusive measurements of the $p d \rightarrow{ }^{3} \mathrm{He} \mathrm{X}$ reaction by Abashian, Booth and Crowe [1]. Subsequent bubble-chamber [2,3] and single-arm magnetic spectrometer measurements [4-11] suggested this enhancement to be correlated with the production of an isoscalar pion pair in fusion reactions to light nuclei. Its explanation has been a puzzle since more than 50 years. Therefore it has been named just after the initials of those authors, who first observed this effect.

In recent exclusive and kinematically complete measurements of the $p n \rightarrow d \pi^{0} \pi^{0}$ reaction it has been demonstrated [12-14] that the ABC effect in this basic double-pionic fusion reaction is correlated with a narrow structure in the total cross section with quantum numbers $I\left(J^{P}\right)=0\left(3^{+}\right)$, a mass of 2.37 $\mathrm{GeV}$ and a width of about $70 \mathrm{MeV}$. The mass is about $90 \mathrm{MeV}$ below $2 m_{\Delta}$, the mass of a $\Delta \Delta$ system and the width is three times narrower than expected from a conventional $t$-channel $\Delta \Delta$ process.

On the contrary the basic isovector fusion process $p p \rightarrow d \pi^{+} \pi^{0}$ exhibits neither an $\mathrm{ABC}$ effect nor a narrow resonance structure $[14,15]$ in this particular energy region - in agreement with the observations in all other two-pion channels of $p p$ initiated reactions [16-27], i.e. of isovector reactions.

\footnotetext{
a e-mail: clement@pit.physik.uni-tuebingen.de
} 


\section{Exclusive and Kinematically Complete Experiments}

In an effort to solve this long-standing problem by exclusive and kinematically complete high-statistics experiments, we have measured the fusion reactions to $\mathrm{d},{ }^{3} \mathrm{He}$ and ${ }^{4} \mathrm{He}$ with WASA at COSY. These measurements cover the full energy region, where the $\mathrm{ABC}$ effect has been observed previously in inclusive reactions. They complement the systematic two-pion production measurements carried out at PROMICE/WASA [17-19], CELSIUS/WASA [13,20-23, 28, 29], COSY-TOF [24, 25], COSYMOMO [30], WASA-at-COSY [26,31,32] and COSY-ANKE [27].

The bulk of these experiments has been performed with the WASA detector featuring a solid angle coverage of nearly $4 \pi$, windowless hydrogen and deuterium pellet target systems $[33,34]$ as well as a reliable particle identification for $\gamma, \pi, p, n, d,{ }^{3} \mathrm{He}$ and ${ }^{4} \mathrm{He}$ ejectiles.

Measurements at WASA have been performed by detection of all ejectiles of an event (with the exception of spectator nucleons) allowing thus kinematic fits with several overconstraints in the data analysis. The $p n$ initiated reactions have been studied via the quasifree $p d$ process with a spectator proton resulting from the target deuteron. By use of the Fermi motion of the active nucleon in the deuteron target the energy dependence of $p n$ and $p p$ initiated reactions could be measured over a range of more than $100 \mathrm{MeV}$ with just a single beam energy.

\section{Discussion of Results}

From these measurements total as well as differential cross sections have been obtained over the full energy region, where the $\mathrm{ABC}$ effect has been found previously.

As an example we show in Fig. 1 the distribution of the $\pi^{0} \pi^{0}$ invariant mass for the double pionic fusions to $\mathrm{d}$ and ${ }^{4} \mathrm{He}$. In both cases we observe a pronounced $\mathrm{ABC}$ effect, i.e. low-mass enhancement - similar to the situation in the $p d \rightarrow{ }^{3} \mathrm{He} \pi^{0} \pi^{0}$ reaction [28]. This experimental finding strongly contravenes conventional calculations based on the mutual excitation of the two colliding nucleons to their $4(1232)$ state by $t$-channel meson exchange, which rather predict a gentle two-hump structure relative to phase space (dotted lines in Fig. 1) [12,22,31]. Such conventional calculations give a good account for the data for $p p$ initiated two-pion production $[15,22,26]$. Hence it comes as a surprise that they obviously do not work for $p n$ initiated two-pion production - in particular fusion processes.

The basic double-pionic fusion to deuterium features three different reaction channels:

- $p n \rightarrow d \pi^{0} \pi^{0}$, which is purely isoscalar

- $p p \rightarrow d \pi^{+} \pi^{0}$, which is purely isovector, and

- $p n \rightarrow d \pi^{+} \pi^{-}$, which contains both isoscalar and isovector contributions.

They are connected by the following isospin relation [35] for their total cross sections:

$$
\sigma\left(p n \rightarrow d \pi^{+} \pi^{-}\right)=2 \sigma\left(p n \rightarrow d \pi^{0} \pi^{0}\right)+\frac{1}{2} \sigma\left(p p \rightarrow d \pi^{+} \pi^{0}\right),
$$

which allows for a cross check on the internal consistency of measurements.

Based on our measurements of all three reactions we have carried out an isospin decomposition of the isospin-mixed $p n \rightarrow d \pi^{+} \pi^{-}$reaction by use of both the isovector $p p \rightarrow d \pi^{+} \pi^{0}$ and the isoscalar $p n \rightarrow d \pi^{0} \pi^{0}$ channel. We find internal consistency of the measurements within their uncertainties. The result of the isospin decomposition is shown in Fig. 2 [14,36].

\subsection{Isovector Double-Pionic Fusion}

The purely isovector $p p \rightarrow d \pi^{+} \pi^{0}$ reaction exhibits no ABC effect in the $\pi^{+} \pi^{0}$ invariant mass distribution, which is plausible, since the isovector $\pi^{+} \pi^{0}$ pair needs to be in relative $p$-wave due to Bose symmetry. Accordingly the low mass region appears to be suppressed relative to phase space rather than enhanced. This is borne out in the data and properly reproduced by conventional calculations of the $t$-channel $\Delta \Delta$ process $[14,15]$. In the energy region of interest the total cross section rises smoothly (black filled circles in Fig. 2) - again in accord with the conventional calculations. 

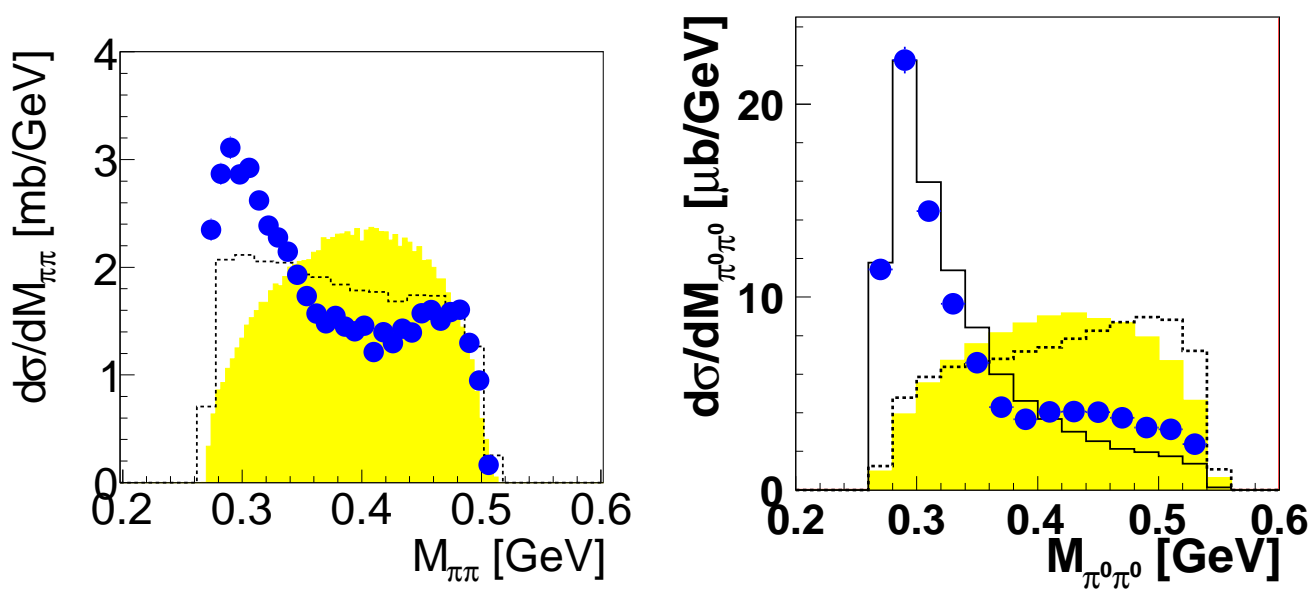

Fig. 1. Distributions of the $\pi^{0} \pi^{0}$ invariant mass $M_{\pi^{0} \pi^{0}}$ observed in the reactions $p n \rightarrow d \pi^{0} \pi^{0}$ (left) and $d d \rightarrow{ }^{4} \mathrm{He} \pi^{0} \pi^{0}$ (right) at incident beam energies of $1.1 \mathrm{GeV}$. The shaded areas represent the phase-space distributions. Dotted lines give $t$-channel $\Delta \Delta$ calculations, whereas the solid line in the right figure shows a calculation of a $p n s$-channel resonance for illustration $[12,31,32]$.

\subsection{Isoscalar Double-Pionic Fusion}

In contrast, the purely isoscalar fusion reaction $p n \rightarrow d \pi^{0} \pi^{0}$ does not behave as expected from conventional reaction dynamics. It rather exhibits a narrow resonance structure in the total cross section (blue filled circles in Fig. 2), which is correlated with the appearance of the ABC effect in the $\pi^{0} \pi^{0}$ invariant mass spectrum (Fig. 1) [12-14]. Its peak energy is about $90 \mathrm{MeV}$ below the nominal $\Delta \Delta$ threshold of $2 m_{\Delta}$ and its width of only $70 \mathrm{MeV}$ is much less than the width of $2 \Gamma_{\Delta}$ expected from the conventional $t$-channel $\Delta \Delta$ process. From the angular distributions the quantum numbers $I\left(J^{P}\right)=0\left(3^{+}\right)$ have been assigned to this structure [12]. From Dalitz plots we may deduce that this state decays first into a $\Delta \Delta$ system before it reaches the $d \pi^{0} \pi^{0}$ final system.

Such a kind of a dibaryonic state has actually been predicted in some theoretical models, see $e . g$. $[37,38]$. At present no conventional process is known, which could at least qualitatively explain this phenomenon.

Note that this reaction constitutes the only two-pion production channel in $N N$ collisions, which is purely isoscalar. Hence exotic processes in the isoscalar channel are likely to be seen best in this particular reaction without being buried underneath large isovector contributions.

In the double-pionic fusion reaction $d d \rightarrow{ }^{4} \mathrm{He} \pi^{0} \pi^{0}$ again the ABC effect is observed to be correlated with the appearance of a resonance-like structure in the total cross section at the same excess energy, however, with an increased width due to Fermi motion in initial and final nuclei as well as due to collision damping, see Fig. 3 [31,32]. From this we conclude that this resonance structure obviously is strong enough to survive even in light nuclei.

The energy dependence of the reaction $p d \rightarrow{ }^{3} \mathrm{He} \pi \pi$ is under investigation. Preliminary results [39, $40]$ indicate a similar trend as observed for the ${ }^{4} \mathrm{He}$ case.

\section{Conclusions and Outlook}

Based on the large amount of meanwhile available data we observe a scenario, which is surprisingly different, whether the two-pion production is initiated by isovector or isoscalar $N N$ collisions. Whereas all $p p$, i.e. isovector initiated reaction channels may be well understood by conventional $t$-channel processes, the two-pion production initiated by isoscalar $p n$ collisions is not understood by such processes alone. The outstanding finding here is that the $\mathrm{ABC}$ effect known for more than fifty years is strictly 


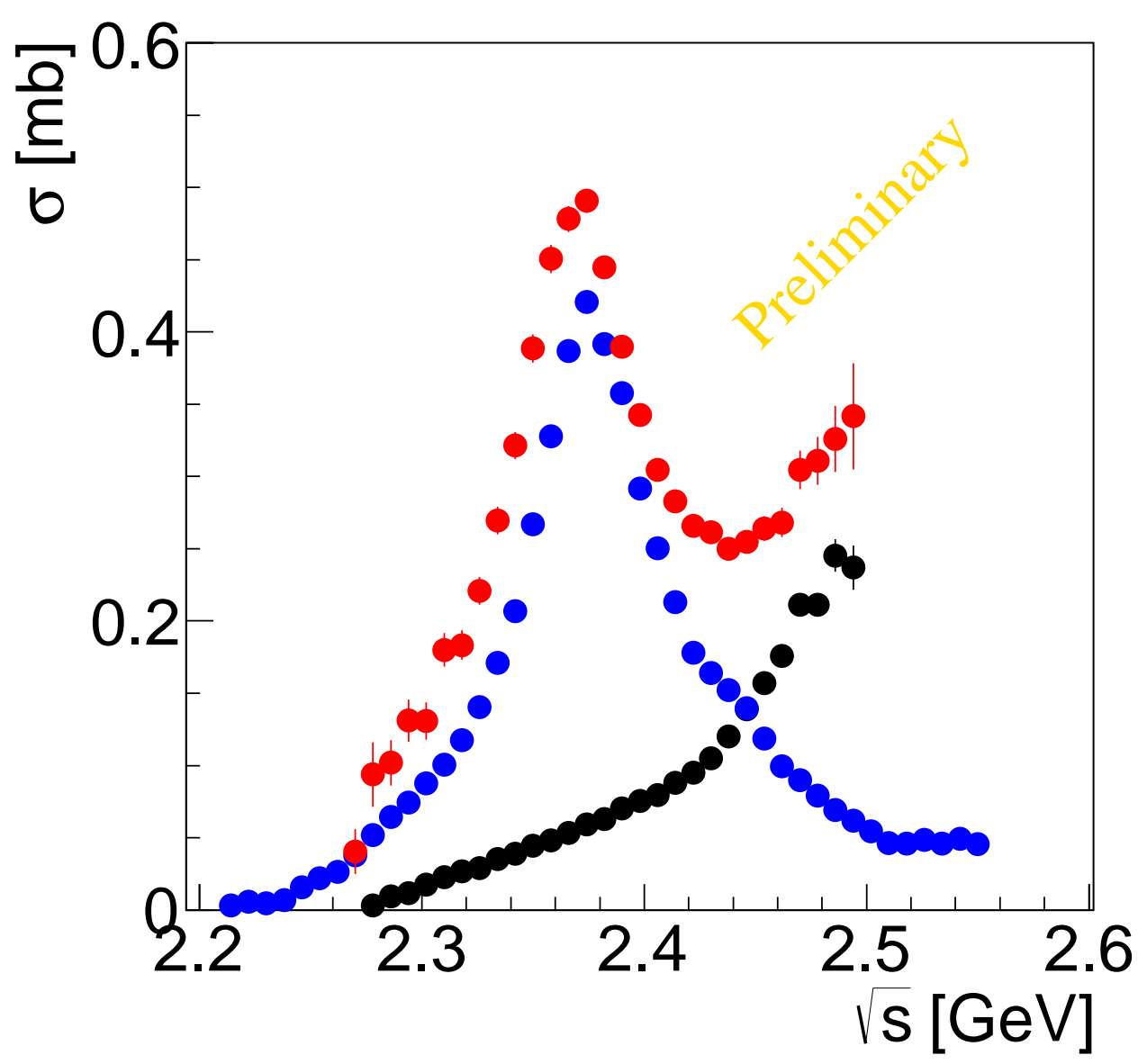

Fig. 2. Preliminary results for the total cross sections of the basic double-pionic fusion reactions $p N \rightarrow d \pi \pi$ of different isospin systems in dependence of the center-of-mass energy $\sqrt{s}$ from threshold $(\sqrt{s}=2.15 \mathrm{GeV})$ up to $2.6 \mathrm{GeV}$. The data for the isospin mixed reaction $p n \rightarrow d \pi^{+} \pi^{-}$are shown by the red full circles. The data for its isovector part given by half the cross section of the $p p \rightarrow d \pi^{+} \pi^{0}$ reaction are plotted by the black filled circles, whereas the data for its isoscalar part given by twice the cross section of the $p n \rightarrow d \pi^{0} \pi^{0}$ reaction are shown by the blue filled circles. From Ref. [36].

correlated with a narrow resonance-like structure in the total cross section. For the purely isoscalar $p n \rightarrow d \pi^{0} \pi^{0}$ reaction this phenomenon - interpreted as $s$-channel resonance with $I\left(J^{P}\right)=0\left(3^{+}\right)-$ comprises most of the measured cross section. The same situation is found for the double-pionic fusion to the nuclei ${ }^{3} \mathrm{He}$ and ${ }^{4} \mathrm{He}$. If true then this isoscalar $p n$ resonance structure is obviously strong enough to survive even in nuclei.

In forthcoming investigations signals of such a resonance will be searched for in the reaction channels $p n \rightarrow p n \pi^{0} \pi^{0}[41]$ and especially in $p n$ elastic scattering. The $p n \rightarrow p p \pi^{0} \pi^{-}$reaction, where also the resonance should show up in principle, is currently under investigation [16]. Also singlepion production channels could in principle contain effects from this resonance. However, the large conventional cross sections in these channels make it very difficult to search for the resonance there. 


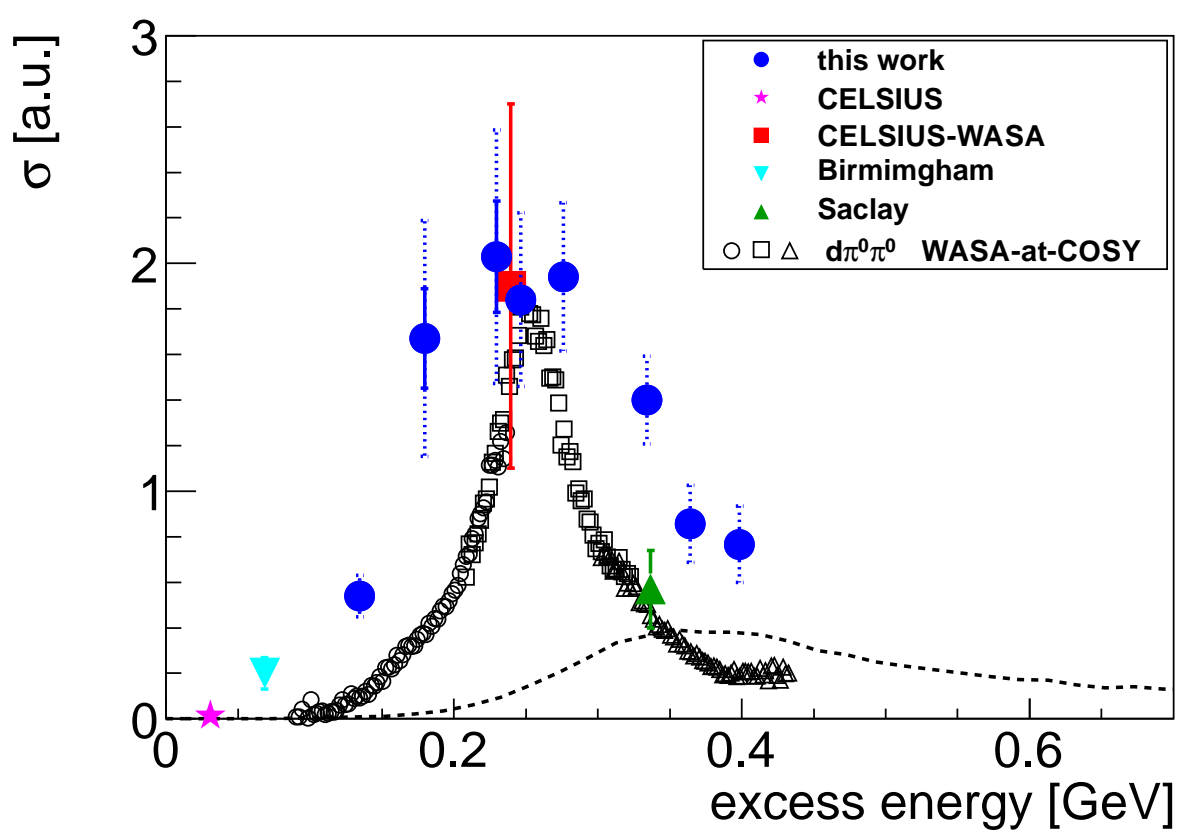

Fig. 3. Total cross sections of the $p n \rightarrow d \pi^{0} \pi^{0}$ (black symbols) and $d d \rightarrow{ }^{4} \mathrm{He} \pi^{0} \pi^{0}$ reactions (colored symbols) in dependence of the excess energy above threshold. Both cross sections are given in relative units and scaled such as to have comparable heights in the peak cross section. The WASA-at-COSY results (solid circles) are compared to results from a previous exclusive measurement at CELSIUS/WASA [29] (square) as well as inclusive measurements at CELSIUS [42] (star), Birmingham [43] (inverted triangle) and Saclay [7] (triangle). The dotted line shows the energy dependence of the conventional $t$-channel $\Delta \Delta$ process, arbitrarily scaled in height.

\section{Acknowledgments}

We acknowledge valuable discussions with C. Hanhart, E. Oset, A. Sibirtsev, F. Wang and C. Wilkin on this issue. This work has been supported by BMBF (06TU9193) and Forschungszentrum Jülich (COSY-FFE).

\section{References}

1. N. E. Booth, A. Abashian, K. M. Crowe, Phys. Rev. Lett. 7 (1961) 35; 5 (1960) 258; Phys. Rev. 132 (1963) $2296 \mathrm{ff}$

2. I. Bar-Nir et al., Nucl. Phys. B 54 (1973) 17

3. A. Abdivaliev et al., Sov. J. Nucl. Phys. 29 (1979) 796

4. R. J. Homer et al., Phys. Rev. Lett. 9 (1964) 72

5. J. H.Hall et al., Nucl. Phys. B 12 (1969) 573

6. J. Banaigs et al., Nucl. Phys. B 67 (1973) 1

7. J. Banaigs et al., Nucl. Phys. B 105 (1976) 52

8. F.Plouin et al., Nucl. Phys. A 302 (1978) 413

9. F. Plouin, P. Fleury, C. Wilkin, Phys. Rev. Lett. 65 (1990) 690

10. R. Wurzinger et al., Phys. Lett. B 445 (1999) 423

11. for a review see A. Codino and F. Plouin, LNS/Ph/94-06

12. P. Adlarson et al., Phys. Rev. Lett. 106 (2011) 202302

13. M. Bashkanov et al., Phys. Rev. Lett. 102 (2009) 052301 
14. M. Bashkanov et al., contribution to this conference and to be published

15. F. Kren et al., Phys. Lett. B 684 (2010) 110 and B 702 (2011) 312; arXiv:0910.0995v2 [nucl-ex]

16. T. Skorodko et al., contribution to this conference and to be published

17. J. Johanson et al., Nucl. Phys. A 712 (2002) 75

18. W. Brodowski et al., Phys. Rev. Lett. 88 (2002) 192301

19. J. Pätzold et al., Phys. Rev. C 67 (2003) 052202 (2005) 294

20. T. Skorodko, et al., Eur. Phys. J. A 35 (2008) 317

21. T. Skorodko, et al., Phys. Lett. B 679 (2009) 30

22. T. Skorodko et al., Phys. Lett. B 695 (2011) 115

23. T. Skorodko et al., Eur. Phs. J. A 47 (2011) 108

24. S. Abd El-Bary et al., Eur. Phys. J. A 37 (2008) 267

25. S. Abd El-Samad et al., Eur. Phys. J. A 42 (2009) 159

26. P. Adlarson et al., Phys. Lett. B 706 (2011) 256; arXiv:1107.0879 [hep-ex]

27. S. Dymov et al., Phys. Rev. Lett. 102 (2009) 192301

28. M. Bashkanov et al., Phys. Lett. B 637 (2006) 223

29. S. Keleta et al., Nucl. Phys. A 825 (2009) 71

30. F. Bellemann et al., Phys. Rev. C 60 (1999) 061002

31. A. Pricking, PhD thesis, Univ. Tübingen 2011; http://tobias-lib.unituebingen.de/volltexte/2011/5695/pdf/ThesisFinal.pdf

32. P. Adlarson et al., submitted for publication; arXiv:1206.6337 [nucl-ex]

33. Ch. Bargholtz et al., Nucl. Instrum. Methods A 547 (2005) 294

34. H. H. Adam et al., arXiv:nucl-ex/0411038 (2004)

35. J. Bystricky it et al., J. Physique 48 (1987) 1901 and references therein

36. COSY Annual Report highlights 2012

37. J. Ping et al., Phys. Rev. C 79 (2009) 024001 and references therein

38. P. J. Mulders, A. Th. Aerts and J. J. de Swart, Phys. Rev. D 21 (1980) 2653

39. E. Perez del Rio et al., contribution to this conference

40. E. Perez del Rio et al., contribution to the Etaprime Workshop, Aachen 2011; arXiv:1204.5509 [nucl-ex] (2012)

41. G. Fäldt and C. Wilkin, Phys. Lett. B 701, (2011) 619

42. Ch. Bargholtz et al., Phys. Lett. B 398 (1997) 264

43. K. R. Chapman et al., Phys. Lett. 21 (1966) 465 